\title{
PEARLS home based treatment significantly improves depression, dysthymia, and health related quality of life in older people
}

Ciechanowski P, Wagner E, Schmaling K, et al. Community-integrated home-based depression treatment in older adults. A randomized controlled trial. JAMA 2004;291:1569-77.

How effective is a home based treatment for minor depression and dysthymia in older people with chronic illnesses?

\section{METHODS}

$\square$

Design: Randomised controlled trial.

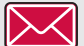

Allocation: Concealed.

Blinding: Assessors blinded to treatment.

Follow up period: 12 months.

Setting: Seattle, Washington, USA; January 2000 to May 2003

Patients: 138 adults with a mean age of 73 years (79\% women) Participants had minor depression $(51 \%)$ or dysthymia $(49 \%)$ and a mean of 4.6 chronic medical ailments. $72 \%$ of participants also lived alone. People with major depression, bipolar disorder, psychosis, substance abuse, or cognitive impairment were excluded.

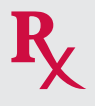

Intervention: Participants were randomly assigned to usual care or the home based intervention: Program Encourage Active, Rewarding Lives for Seniors (PEARLS). PEARLS involved eight 50 minute problem solving treatment sessions with social and physical activities, over 19 weeks; these sessions were modified to increase social and physical activities. Physical activation began at session 3 or 4 and aimed to encourage regular moderate physical activity for at least 30 minutes, five days a week. Social activation aimed to increase participants' interactions outside the home, with group activities encouraging peer support given highest priority. Each session included selecting pleasant activities to engage in before the next session (from a list of 250).

Outcomes: Improvement of depressive symptoms: Hopkins Symptom Checklist 20, (range 1-4, with 1.72 indicating major depression); improvement in health related quality of life (Functional Assessment of Cancer Therapy Scale-General). Patient follow up: $92 \%$ at 12 months.

\section{MAIN RESULTS}

At 12 months, PEARLS significantly reduced depressive symptoms compared with usual care $(50 \%$ or greater reduction in depressive

For correspondence: Paul Ciechanowski, Department of Psychiatry and Behavioural Sciences, University of Washington School of Medicine, NE Pacific, Seattle, WA 356560, USA; pavelcie@u.washington.edu

Sources of funding: the Prevention Research Centers Program of the Centers for Disease Control and Prevention and University of Washington Health Promotion Research Center. symptoms: $43 \%$ v 15\%; OR 5.21; 95\% CI 2.01 to 13.49). PEARLS significantly increased complete remission compared with usual care (36\% $v 12 \%$, OR $4.96 ; 95 \%$ CI 1.79 to 13.72 ). PEARLS significantly improved health related quality of life in the domains of functional wellbeing $(p=0.001)$ and emotional wellbeing $(p=0.048)$ compared with usual care. There were no significant differences between groups in social and physical wellbeing.

\section{CONCLUSIONS}

The PEARLS home based treatment significantly reduces symptoms of depression and improves health related quality of life for elderly people with minor depression and dysthymia.

\section{Commentary}

A fter dementia, depression is the most common psychiatric disorder in the elderly and the two disorders are often comorbid. However, the under diagnosis of depressive syndromes and their inadequate treatment is endemic. Many explanations have been put forward in an attempt to understand the reasons for this state of affairs, such as an inadequate knowledge base, ageism, patient somatisation and difficulty in symptom attribution in the presence of medical illness. To make matters worse, there are a large number of people who have subsyndromal depressions (dysthymia and minor depression). There is very little that we know about the course and impact of these disorders. A four year study of Health Maintenance Organization (HMO) enrolees aged 65 years or older found persistent depressive symptoms to be common and was associated with higher healthcare costs. ${ }^{1}$ We know even less about the impact of pharmacological or non-pharmacological interventions in this population. In this context the paper by Ciechanowski et al adds to our evidence base. It gives us a method of identifying cases in the community with minor depression or dysthymia. It supports the benefits of a case management system and the efficacy of a psychosocial intervention. Case managers have been found in primary care settings to be effective in promoting patient adherence to medications. ${ }^{2}{ }^{3}$ Adherence is an important issue because it has been shown that $20 \%$ of patients may not fill an initial prescription. ${ }^{4}$ Although the methods and interventions used in this study are not readily applicable to the individual practitioner's office, it is something that HMOs and other large insurers should consider implementing since it has the potential to reduce their costs in the long run.

Professor Ashok Raj MD USF College of Medicine, Tampa, Florida, USA

1 Unutzer J, Patrick DL, Simon G, et al. Depressive symptoms and the cost of health services in $\mathrm{HMO}$ patients aged 65 years and older. JAMA 1997:277:1618-23.

2 Unutzer J, Katon W, Callahan CM, et al. Collaborative care management of late life depression in the primary care setting: a randomized controlled trial. JAMA 2002;288:2836-45.

3 Bruce ML, Ten Have TR, Reynolds CF III. Reducing suicidal ideation and depressive symptoms in depressed older primary care patients: a randomized controlled trial. JAMA 2004:291:1081-91

4 Burns JM, Sneddon I, Lovell M, et al. Elderly patients and their medication: a post discharge follow up study. Age Ageing 1992;21:178-81. 